\title{
Letters and comments
}

Contributors to this section are asked to make their comments brief and to the point. Letters should comply with the Notice printed on the inside back cover. Tables and figures should only be included if absolutely essential and no more than five references should be given. The Editor reserves the right to shorten letters and to subedit contributions to ensure clarity.

Response to paper by ML Rogers et al.

\section{Surgical treatment of para-oesophageal hiatal hernia}

Ann R Coll Surg Engl 2001; 83: 394-8

\section{F. Fausto Palazzo, Majid Hashemi, John Cochrane}

Department of Surgery, Whittington Hospital, London, UK

\begin{abstract}
$\mathrm{A}$ lthough para-oesophageal hernias may be complicated by potentially fatal complications, it is not imperative that all are immediately treated surgically. Treacy and Jamieson demonstrated, in a series of 52 elective para-oesophageal hernia presentations, that the adoption of a selective approach allowed them to avoid surgery in 11 of 52 cases. ${ }^{1}$ In another series, Allen et al. reported only 3 cases of gastric strangulation in 735 patient-years follow-up. ${ }^{2}$

The true incidence of recurrence following paraoesophageal hernia repair is difficult to establish since most recurrences remain asymptomatic and most series lack objective radiological or endoscopic longterm follow-up. Various measures are known to reduce the risk of recurrence: a crural repair is an integral part of para-oesophageal hernia repair since recurrence appears to be guaranteed in its absence; ${ }^{3}$ the approach to this crural repair needs to ensure good tension free apposition of what are thin and attenuated fibres. The better long-term results after thoracotomy and laparotomy when compared with laparoscopy may reflect the effectiveness and durability of the crural repair.

Complications of deliberate intrathoracic placement of fundoplications are well recognised, ${ }^{4}$ but it does not appear that late asymptomatic re-herniations after para-oesophageal hernia repair are subject to the same risks. Close follow-up with treatment of symptomatic patients only appears to be a satisfactory approach..$^{5,6}$
\end{abstract}

\section{References}

1. Treacy PJ, Jamieson GG. An approach to the management of paraoesophageal hiatus hernia. Aust N Z J Surg 1987; 57: 813-7.

2. Allen MS, Trastek VF, Deschamps C, Pariolero PC. Intrathoracic stomach. Presentation and results of operation. J Thorac Cardiovasc Surg 1993; 105: 253-8.

3. Loustarinen M, Rantalainen M, Helve O, Reinikainen P, Isolauri J. Late results of paraoesophageal hiatus hernia repair with fundoplication. Br J Surg 1998; 85: 272-5.

4. Richardson JD, Larson GM, Polk HC. Intrathoracic fundoplication for shortened esophagus: treacherous solution to a challenging problem. Am J Surg 1982; 143: 29-35.

5. Hashemi M, Peters JH, DeMeester TR, Huprich J, Quek M, Hagen $\mathrm{JH}$ et al. Laparoscopic repair of paraoesophageal hiatal hernias: objective evaluation reveals high recurrence rates. J Am Coll Surg 2000; 190: 553-60.

6. Soper NJ, Dunnegan D. Anatomic fundoplication failure after laparoscopic antireflux surgery. Ann Surg 1999; 229: 669-77.

Correspondence to: Mr F. Fausto Palazzo MS FRCS, SpR in Surgery, John Radcliffe Hospital, Oxford, UK

E-mail: ffpalazzo@aol.com

\section{Surgical site identification - think before you print!}

\section{RW Goyal, ME Lovell}

Department of Orthopaedics and Trauma, South Manchester University Hospital Trust, Manchester, UK

The implications of 'wrong-site' surgery can be occurrences include pre-operative surgical site identification via an arrow or an ' $\mathrm{X}$, ${ }^{1}$ whole others use the word ' $\mathrm{No}^{\prime}$ to 


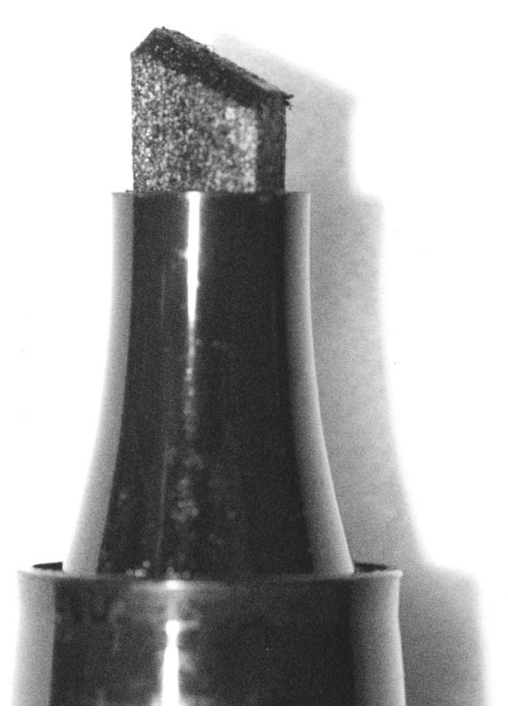

Figure 1 The profile of the pen demonstrating the 'sharpness' of its tip.

mark the limb or site that should not be operated upon. ${ }^{2,3}$ Certain centres employ a checklist policy. ${ }^{4}$

Despite the wide-spread use of surgical site identification with pen markers, a number of complications have been reported. Contact dermatitis, ${ }^{5,6}$ secondary to the constituents of the pen-ink, is well described. In addition, there are reports of patients crossing their legs before the ink is dry and producing an identical mark on the contralateral limb, thus subverting the intended effect of limb marking. ${ }^{7}$

We wish to report that selection of a suitable pen should also be borne in mind. This simple fact must not be overlooked considering the range of marker pens that can be freely seen on any hospital ward. One patient of ours, due to have a total knee replacement, was found by the nursing staff to be bleeding from the anteromedial surface of her leg shortly after an arrow was placed. The profile of the pen was examined (Fig. 1).

This complication can be avoided by ensuring that the 'pen-mark' is placed in areas with satisfactory soft tissue coverage (avoid marking skin where bone is subcutaneous), as well as ensuring that the profile of the pen-tip is soft or brush-like. Vigilance is always required in the elderly where skin may be fragile.

\section{References}

1. American Academy of Orthopedic Surgeons. Report of the Task Force on Wrong-site Surgery. Accessed October 30, 2001. http:/www.aaos.org/wordhtml/mede/tasksite/html

2. Lubicky JP. Wrong-site surgery. J Bone Joint Surg Am 1998; 80: 1398.

3. Cowell HR. Wrong-site surgery. J Bone Joint Surg Am 1998; 80: 463.

4. Anon. Sentinel events: approaches to error reduction and prevention. It Comm J Qual Improv 1998; 24: 175-86.

5. Hamilton TK, Zug KA. Triethanolamine allergy inadvertently discovered from a fluorescent marking pen. Am J Contact Dermat 1996; 7: 164-5.

6. Hagdrup H, Egsgaard H, Carlson L, Anderson KE. Contact allergy to 2-hydroxy-5-tert-butyl benzylalcohol and 2,6-bis-(hydroxymethyl)-4tert-butylphenol, components of a phenolic resin used in marking pens. Contact Dermatitis 1994; 31: 154-6.

7. Anon. Position Paper on Wrong-sided Surgery in Orthopaedics. Winnipeg, Manitoba: Canadian Orthopedic Association Committee on Practice and Economics, 1994.

Correspondence to: Mr RW Goyal, Flat 1 Denefield Place, 5

Sandwich Road, Manchester M30 9HD, UK.

E-mail: ravi.goyal@ukgateway.net

Response to paper by CM Gupte et al

\section{The Internet - friend or foe? A question- naire study of orthopaedic out-patients}

\author{
Ann R Coll Surg Engl 2002; 84: 187-92
}

Letter 1

MD Gough, CJ Stoddard, R Ackroyd

Department of Surgical and Anaesthetic Sciences, Royal Hallamshire Hospital, Sheffield, UK

$W^{2}$ e have performed a similar questionnaire study looking at information given to patients with Barrett's oesophagus. We found that information given to these patients was often less than ideal. Of our patients, $33.8 \%$ had personal access to the Internet and $53.8 \%$ of patients would use the Internet for medical information if they had access. The average age of those prepared to use the Internet was over 10 years less than those who refused to use the Internet. Several of our respondents that had already used the Internet stated that the sites found were very technical and not aimed at the average patient.

We believe that the medical profession should encourage Internet usage, but patients should be guided to sites either produced or vetted by the practitioner. Those not able to use the Internet should not be forgotten, and adequate information should still be provided in a suitable format.

Correspondence to:

Mr R Ackroyd, Department of Surgery, K Floor, Royal Hallamshire Hospital, Glossop Road, Sheffield S10 2JF, UK Tel/Fax: +44 114226 1398; E-mail: r.ackroyd@sheffield.ac.uk 
Response from authors

\section{Gupte, ANA Hassan, ID McDermott, RD Thomas}

Mechanical Engineering Department, Imperial College of Science, Technology and Medicine, London, UK

Several studies, including your own, indicate the $\mathcal{S}$ willingness of patients to seek medical information over the Internet. We did not directly question patients or surgeons as to whether information obtained from the Internet helped or hindered the consultation. However, nearly $24 \%$ of patients reported that they found information from the web sites confusing, and it is likely that this hindered the consultation.

We agree that peer review of medical web sites remains an issue of some concern. With regard to surgery, perhaps the Joint Colleges of Surgeons should set up a working party to address this issue.

Correspondence to: Mr CM Gupte MA BM BCh MRCS,

Research Fellow, Mechanical Engineering Department, Imperial College of Science, Technology and Medicine, Exhibition Road, London SW7 2BX, UK.

Tel: +44 207594 7062; Fax: +44 207823 8845;

E-mail: c.gupte@ic.ac.uk

Letter 2

\section{RR Brown}

Department of Orthopaedic Surgery, The Middlesex Hospital, London, UK

$\mathrm{T}^{\mathrm{s}}$ This was a very informative article, which helps to quantify an activity which will alter surgical practice in all surgical specialities. We were interested to see that $12.3 \%$ of the patients in the study at a district general hospital had researched their particular condition on the Internet. In December 2000 and January 2001 we conducted a multicentre study of Internet usage by 350 orthopaedic patients, which showed a mean usage of $8.3 \%{ }^{1}$ When was the data collected? Does this increased rate of usage help to confirm the trend of increasing Internet usage by patients?

We also found that Internet usage was higher in elective clinics and in tertiary referral clinics. Internet use was $22 \%$ by patients attending a tertiary referral clinic, compared to $9 \%(P=0.01)$ at a district general hospital.

In our study, we were concerned to find that only $20 \%$ of Internet sites visited by patients were peer reviewed, casting a question on the quality of information. Did the authors have any way to assess whether the obtained medical information helped or hindered the surgical consultation?

\section{Reference}

1. Wright JED, Brown RR, Chadwick C, Karadagalis D. The use of the Internet by orthopaedic outpatients. J Bone Joint Surg Br 2001; 83: 1096-7.

Correspondence to: Mr RR Brown MA FRCS FRCS(Orth), Specialist Registrar, Department of Orthopaedic Surgery, The Middlesex Hospital, Mortimer Street, London W1N 8AA, UK

\section{Response from authors}

\section{Gupte, ANA Hassan, ID McDermott, RD Thomas}

\section{Mechanical Engineering Department, Imperial College of Science, Technology and Medicine, London, UK}

$\mathrm{O}$ ur study was conducted at a similar time during January 2001. Whilst it is unclear whether the difference of $12.3 \%$ and $8.3 \%$ between our studies is statistically significant, there may be several reasons underlying the difference in usage. It is possible that the demographics between the two populations studied differed significantly, and this was reflected by the usage rates. For example, if the population we studied was significantly younger, this may have resulted in an increased rate of Internet usage in our study. Our studies also used different questionnaires, which may have influenced the responses.

As the studies were conducted at approximately similar times, we cannot conclude that the rate of use is increasing. However, the finding in our study that younger patients were more likely to use the Internet would indicate that this activity will increase in the future as this younger population ages. A follow-up study is planned in the same centre. We also found that elective patients who were repeat visitors were more likely to use the Internet for a medical condition than those who were first-time referrals from casualty (odds ratio, 3.2; 95\% CI, 1.9-4). This may indicate that as patients become more familiar with their condition, they are more likely to surf the web for further information.

We did not directly question patients or surgeons as to whether information obtained from the Internet helped or hindered the consultation. However, nearly $24 \%$ of patients reported that they found information 
from the web sites confusing, and it is likely that this hindered the consultation.

We agree with you that peer review of medical web sites remains an issue of some concern. With regard to surgery, perhaps the Joint Colleges of Surgeons should set up a working party to address this issue.

Correspondence to: Mr CM Gupte MA BM BCh MRCS,

Research Fellow, Mechanical Engineering Department, Imperial College of Science, Technology and Medicine, Exhibition Road, London SW7 2BX, UK

Tel: +44 207594 7062; Fax: +44 207823 8845;

E-mail: c.gupte@ic.ac.uk

\section{Response to paper by A Unwin}

\section{Arthroscopy of the knee joint}

Ann R Coll Surg Engl 2002; 84: 137-9

\section{Adrian C Fairbank \\ Trauma and Orthopaedic Department, St George's Hospital, London, UK}

\begin{abstract}
rthroscopy of the knee has a very low infection Arate. I am, therefore, concerned that the author has stated that he would give broad-spectrum antibiotics intravenously at induction. Given the very low infection rate associated with the procedure, I would consider this unnecessary.
\end{abstract}

Correspondence to: Mr Adrian C Fairbank MA FRCS(Orth), Consultant Orthopaedic Surgeon, Trauma and Orthopaedic Department, St George's Hospital, Blackshaw Road, London SW17 0QT, UK. Fax: +44 2087253610

\section{Response from author}

\section{A Unwin}

The Windsor Orthopaedic Clinic, Windsor, Berkshire, UK

$\mathrm{T}$ his Technical Tutorial was a synopsis of my current method of arthroscopy and this includes the administration of broad-spectrum antibiotics intravenously at induction. I agree that the infection rate after arthroscopy is low, but the potential for disaster with such an infection is high. Many arthroscopy operating lists are high volume and turnover and especially where a laminar flow or similar theatre facility is unavailable there is a potential for infection. The use of broadspectrum antibiotics as prophylaxis has not been shown to increase resistance.

It would be very difficult to design a study to compare the infection rate after arthroscopy with or without antibiotics as the infection rate is low.

I accept that there is a cost implication to my practice, but I feel more secure in administering antibiotics and this is why I included it in my tutorial. Many of the other regimens we use in arthroscopy (e.g. mechanical thrombo-prophylaxis) are also without evidence base, but have a cost implication.

Correspondence to: Mr Andrew Unwin BSc FRCS(Orth), Consultant Orthopaedic Surgeon, The Windsor Orthopaedic Clinic, Phoenix House, Nightingale Walk, Windsor, Berkshire SL4 3HS, UK. Tel: +44 1753 868622; Fax: +44 1753868642

Response to paper by

A McLaren, G Morris-Stiff $\mathcal{E} J$ Casey

\section{Issues of consent in renal transplantation}

Ann R Coll Surg Engl 2001; 83: 343-6

S Sunil, R Austin, S Sinha

Sir Peter Medawar Unit, The Royal Liverpool University Hospital, Liverpool, UK

We agree with the authors that obtaining informed consent for renal transplantation is a complex process and that there are differences of opinion within the transplant community as to the level of risk disclosure required. We noted that consent was obtained by a variety of grades of staff, which included senior house officers and house officers. It was surprising to note that only $13 \%$ discussed malignancy and $25 \%$ did not routinely discuss risks associated with cytomegalovirus infection. There is no mention of primary nonfunction, acute tubular necrosis (ATN), or the need for dialysis and renal transplant biopsies. From the study, it is clear that there is no uniformity in obtaining consent for renal transplantation.

The complexity in obtaining consent for renal transplantation is because of the fact that a multitude of issues need to be discussed in depth with the patient. These should include the donor details, the CMV status of the patient and recipient, operative complications and risks associated with the use of immunosuppressive agents. Patients on the transplant waiting list would have received information at various levels during their assessment for 
renal transplantation. The information is provided by the nephrologists, transplant surgeons and transplant coordinators. There is always a time lag between a patient's clinic visit and the time of admission for a transplant, given the nature of the operation. Also, they are understandably anxious and not in a state of mind to go through the details of a complex consent.

We believe that patients should get the maximum information possible that enables them to make an informed decision. With the introduction of clinical governance, we think that it is time the British Transplantation Society introduces a uniform policy nationally for obtaining informed consent for organ transplantation. We suggest the following to make this complex issue simpler:

1. Standard information leaflets which would give patients information about the various aspects of transplantation including risks and complications related to surgery and immunosuppression, particularly infections and malignancy. These leaflets should be available at renal and transplant clinics, dialysis units as well as GP surgeries.

2. Patients should be aware of the problem of cytomegalovirus infection so that they have the choice to opt for either a CMV-positive or CMVnegative kidney.

3. Only higher surgical trainees (HST) and consultants should obtain consents.

4. A check-list to ensure that all related issues has been discussed with the patient.

Correspondence to: Mr S Sunil, SpR in Transplant Surgery, Sir Peter Medawar Unit, 9C, Link, The Royal Liverpool University Hospital, Prescott Street, Liverpool L7 8XP, UK

Response to paper by B Homer

\section{Breast augmentation should be on the NHS: a discussion of the ethics of rationing}

Ann R Coll Surg Engl 2002; 84: 82-3 Jonathon M Pleat, Chris SJ Dunkin,
Charlotte Davies, Titus Adams

Department of Plastic and Reconstructive Surgery, Stoke Mandeville Hospital, Aylesbury, Buckinghamshire, UK

$\mathrm{W}$ e note with interest the article by Homer in which he succinctly argues the ethical requisites for fuller access to breast augmentation. He is correct to assert that it is ethically wrong to ration this operation within the framework of GMC guidelines.

However, ethical prerogatives can be undermined by entrenched attitudes and financial constraints. First, the absence of 'hard', physical symptoms, compounded by the myriad of evolving psychometric assessment tools, may encourage the conclusion that it is difficult to justify surgery when outcome measures are nebulous. Yet Homer quotes a rigorous paper, supplementing an already substantial body of work, concluding that women with a negative self-perception of small breasts have significant psychological morbidity that is greatly reduced by augmentation. ${ }^{1}$ Not only is the operation efficacious, it is relatively cost-effective. Augmentation produced the second greatest increase in quality adjusted life years (QALYs) for the least expenditure ( $£ 342 / \mathrm{QALY}$ ) from a range of procedures. ${ }^{2}$ The evidence exists, it just seems to be poorly disseminated. Further, the public has misperceptions and suspicions about 'cosmetic surgery'. We have found this with a survey of attitudes of 1000 members of the general public to a variety of plastic and reconstructive surgical operations. Most considered operations with a well-defined physical defect as more 'worthy' than procedures to enhance appearance. This is not surprising given the negative media stance towards the speciality and the poor quality of information from alternative sources such as the Internet. ${ }^{3}$ We suspect that it may take a large-scale and widely advertised study of both benefits to the individual and society (e.g. workplace absenteeism, reduced burden on the medical system) to shift attitudes.

Finally, we disagree with the author's blanket declaration that augmentation should be rationed within the NHS purely on the grounds of psychological distress in conjunction with physical fitness. Up to $5 \%$ of individuals seeking cosmetic surgery have body dysmorphic syndrome. $^{4}$ Their psychological distress involves an excessive pre-occupation with an imagined or slight physical anomaly. Surgery in these patients is contraindicated as it may worsen symptoms. ${ }^{1}$

\section{References}

1. Sarwer D, Wadden T, Whitaker L. An investigation of changes in body image following cosmetic surgery. Plast Reconstr Surg 2002; 109: 363-9.

2. Cole R, Shakespeare P, Hobby J. Measuring outcome in lowpriority plastic surgery patients using quality of life indices. Br J Plast Surg 1994; 47: 117-21.

3. Gordon JB, Barot LR, Fahey AL, Matthews MS. The Internet as a source of information on breast augmentation. Plast Reconstr Surg 2001; 107: 171-6. 
4. Sarwer D, Wadden T, Pertschuk M. Body image dissatisfaction and body dysmorphic disorder in 100 cosmetic surgery patients. Plast Reconstr Surg 1998; 101: 1644-9.

Correspondence to: Mr Jonathon Pleat MA MRCS, Duke of Kent Research Fellow, Smith \& Nephew Research Fellow, Department of Plastic and Reconstructive Surgery, Stoke Mandeville Hospital, Mandeville Road, Aylesbury, Buckinghamshire HP21 8AL, UK. Tel: +44 1296 315909; Fax: +44 870734 8969; E-mail: jonathon.pleat@talk21.com

\section{Response to paper by IC Cameron et al.}

\section{Acute cholecystitis - room for improvement?}

Ann R Coll Surg Engl 2002; 84: 10-3

\section{Kevin B Orr}

\section{Blakehurst, New South Wales, Australia}

Ththe was surprised to see an article ${ }^{1}$ which indicated that there were still surgeons and hospitals in the UK where acute cholecystitis was treated conservatively!

When I left Australia as a young graduate in late 1952 to journey to the UK for surgical training, it was considered by some very influential surgeons in the Australasian College that to take out an acutely inflamed gall bladder was criminal. After a year or two, I obtained a position as registrar with my primary examination at Red Hill County Hospital in Surrey and found that the only way I could obtain a gall bladder to operate on was to do so in the acute stage, owing to a rather selfish senior registrar. The surprise was how well these patients did including one woman who came back from the Continent with a ruptured gall bladder and one must remember that in those days we only had penicillin, streptomycin and chloramphenicol.

When I returned to Australia (Sydney) after 6 years in the UK nothing had happened to disturb my belief that acute gall bladders should be removed forthwith. Others I am sure had the same experience and so Australia passed out of the dark ages and we removed gall bladders in the acute phase.

Not unexpectedly the lesson had to be learnt all over again when the laparoscopic operation came in, but now everything has settled down nicely, though I must say that the removal of an acutely inflamed gall bladder by an inexperienced laparoscopic surgeon would be better done through the small incision open procedure, rather than wait for the patient to settle down and be done laparoscopically.

Cameron and his colleagues at the University Surgical Unit at Hallamshire Hospital, Sheffield give all the correct reasons why the acute gall bladder should be removed on admission, but it is strange that it is almost 50 years since the writer discovered this!

\section{Reference}

1. Cameron IC, Chadwick C, Phillips J, Johnson AG. Acute cholecystitis - room for improvement? Ann R Coll Surg Engl 2002; 84: 10-3.

Correspondence to: Kevin B Orr MBBS FRCS (Engl) FRACS FACS, 42 Castle Street, Blakehurst, New South Wales 2221, Australia

\section{Response from authors}

\section{IC Cameron, AG Johnson}

University Surgical Unit, Royal Hallamshire Hospital, Sheffield, UK

We agree with many of these sentiments that acutely inflamed gallbladders should ideally be removed at the time of initial presentation, provided that this can be done safely. The problem seems to be with the organisation of acute surgical services in the UK at the present time, as high-lighted in a recent survey by ourselves, which was presented at the AUGIS Annual Meeting in 2000. We found that only $11 \%$ of UK general surgeons routinely treat patients with acute cholecystitis by early cholecystectomy. The main reason cited was the lack of availability of experienced surgeons, often due to elective surgical and out-patient commitments. A significant number also commented on the poor provision of daytime emergency theatre time and delays in getting radiological investigations to confirm the diagnosis.

We agree that the weight of evidence favours early cholecystectomy, but unfortunately this is not the practice of the majority of UK surgeons. We are aware this practice is routine in Australasia and in many other European countries, but feel that major organisational change will be needed for it to become standard treatment in the National Health Service under current working conditions.

\footnotetext{
Correspondence to: IC Cameron, Specialist Registrar in Surgery, University Surgical Unit, K Floor, Royal Hallamshire Hospital, Sheffield S10 2JF, UK. Fax: +44 1142713791
} 
Response to paper by A Samsudin et al

\section{Should ICU be part of surgical training?}

Ann R Coll Surg Engl Suppl 2002; 84: 9-11

Neeraj Bhasin, Steve G Dean

Intensive Care Unit, St James's University Hospital, Leeds, UK

W ith regard to this article, we firmly believe that ICU training should be an essential part of a surgical BST training programme, as stated by Prof. Rowlands. ${ }^{1}$ As he writes, training in ICM can only go to improve the quality of patient care by junior staff. ${ }^{1}$ Practical skills that a surgical $\mathrm{SHO}$ would not necessarily gain in other parts of their training (e.g. central venous access, endotracheal intubation, arterial line insertion, use of ionotropes, etc.) are invaluable. The familiarity with these procedures and indications for their use are essential when faced with a critically ill patient. It enables the resident surgical SHO to initiate early critical care treatment and procedures improving patient pathophysiology and outcome at times when senior assistance may not be immediately available.

Due to this multidisciplinary education and integration, there is a continuum of expertise between the wards and the ICU which was stressed in the Department of Health's report Comprehensive Critical Care. This working knowledge of the capabilities of the equipment and monitoring of an ICU enables improved and more effective communication with the critical-care doctors and appropriate use of ICU resources. A period of training in this multidisciplinary setting makes a BST trainee comfortable discussing patients with senior staff and refines the information given and the manner in which it is given. These improved communication skills are also of value with regard to the patient and relatives.

ICU training also has an impact on surgical practice. Having experience of critical care treatment of complications of surgery affects the care taken in consenting patients and their relatives. The moral and ethical dilemmas of withdrawal, futility and appropriateness of treatment are also relevant.

The privileged position of seeing surgery from 'both ends of the table' stresses how the clerking procedure, especially for emergency cases through A\&E, is an opportunity for pre-operative patient optimisation and for appropriate investigations. A few simple steps preoperatively can ease the experience for both the patient and anaesthetist, improving the outcome, and respecting other peoples skills, an essential part of working as a team. ${ }^{3}$

Becoming comfortable with emergency situations and critically ill patients allows for assessment of the patient in a calm, organised, and reasoned manner with the ability to prioritise treatment. Although this is taught on courses, doing this practically on a daily basis it becomes the norm and it may avert admissions to, and enable discharges from, ICU. Again, a point of importance stressed by the Department of Health. ${ }^{2}$

A disadvantage put forward by Samsudin et al. is decreased operating time. ${ }^{1}$ Although this is true, surgical SHOs working in St James's ICU are also included on a part-time basis on the surgical RSO rota, which enables the maintenance of basic surgical skills and a surgical interest. We disagree with the other disadvantage of a lack of research opportunity, ${ }_{1}$ as breaks between shifts on the ICU gives plenty of time to work on a paper or revise for an examination. As well as being able to learn physiology for the MRCS whilst on ICU, the use of this knowledge in everyday practise allows for practical experience rather than merely memorising values or equations. This awareness also extends to pharmacology. Prescribing is outlined as an area of importance in good medical practice by the GMC. ${ }^{3}$ Prescribing in the ICU setting brings a heightened awareness of side effects and interactions, again demanding an overall, multisystem approach to the patient.

We feel that an ICU training post gives a global learning experience which achieves the aims laid out in the Department of Health's report. It allows time to work with enthusiasm, knowing that this opportunity enables trainees to give the best for their patients. ${ }^{2}$

\section{References}

1. Samsudin A, Wheeler JMD, Galland RB et al. Should ICU be part of surgical training? Ann R Coll Surg Engl Suppl 2002; 84: 9-11. Comment by Professor B J Rowlands.

2. Department of Health. Comprehensive Critical Care. Review of Adult Critical Care Services. London: Department of Health, 1999

3. The General Medical Council. Good Medical Practice, 3rd edn. London: The General Medical Council 2001.

Correspondence to: Dr Neeraj Bhasin BSc MBBS, Surgical SHO, Intensive Care Unit, Level 2, Lincoln Wing, St James's University Hospital, Leeds LS9 7TF, UK

\section{Response from authors to}

\section{N. Bhasin \& SG Dean}

\section{MH Lewis}

Department of Surgery, Royal Glamorgan Hospital, Pontypridd $\mathcal{E}$ Rhonda NHS Trust, Llantrisant, UK

The authors do appear to support our view that
SHO/BST rotations onto an ITU is valuable. 
However, the early paragraphs of their letter reflect personal experiences concerning the value of time spent on ITU with no comment with regards to our paper or conclusions. The penultimate paragraph does address our paper and we would reply as follows.

Although we have suggested that there is a disadvantage in taking time away for the practical aspects of surgery by undertaking a stint in ITU, in Leeds the $\mathrm{SHO} / \mathrm{BST}$ ITU attachment is combined with a surgical oncall rota, albeit part-time. Whilst this might maintain some operative experience, the odd night on-call every so often would have questionable value rather than concentrating on the ICU attachment. In addition, we would take issue with the comment that lack of research is a disadvantage whilst attached to ITU. In our paper, we suggest that the BST should find this time valuable, but our findings were that SpRs felt that they were too senior to lose 3 or 6 months from an SpR rotation. We did stress that BST time would be more appropriate attached to an ITU so as to: (i) obtain the experience of an ICU; and (ii) perform research projects either within or without the intensive care environment.

Correspondence to: Mr MH Lewis, Consultant Surgeon, Royal Glamorgan Hospital, Pontypridd \& Rhonda NHS Trust, Ynysmaerdy, Llantrisant CF72 8XR, UK. Tel: +44 1443 443443; Fax: +44 1443443248

\section{Response to paper by}

\section{BA De Souza \& MGD Dickson}

\section{Through the letter box - a traumatic hand injury}

Ann R Coll Surg Engl 2002; 84: 201-2

\section{Anne Seymour \\ Retired Consultant in Accident and Emergency}

Tin note that the potential strategies for preventing such Linjuries listed by the authors are all actions to be taken by the house-holder. One strategy which can be adopted by the leaflet deliverer is to take a short ruler and use this to push the leaflet through the letterbox. This technique was recommended to its helpers by Christian Aid, it is not my own suggestion; but I can testify first-hand that it works!

Correspondence to: Miss Anne Seymour FRCS, 35 The Lonnen, South Shields NE34 8EQ. Tel: +44 1914565134
Response to paper by

$S$ Varshney, M Parimal, CD Johnson

\section{Six-fold suture: wound length ratio for abdominal closure}

Ann R Coll Surg Engl 1999; 81: 333-6.

Rajendra S Rampaul, Vijay Naraynsingh, Dale Maharaj

Department of Surgery, General Hospital, Port of Spain, University of the West Indies, Trinidad, West Indies

W e read with great interest this article by Varshney et al. describing a prospective study into the optimal suture length to wound length ratio (SW:WL) for midline laparotomy closure, using $1 \mathrm{~cm}$ bites in their technique. As the inter-rectus distance is non-uniform as well as wide, thin and weak in obese patients, it is noteworthy that they did not provide data on body mass index and whether this suture always engaged linea alba (as compared to sheath). This is clinically relevant, as some surgeons may routinely engage muscle and sheath or just sheath in their bite and some textbooks recommend a range of bite sizes. ${ }^{1}$ In addition, Campbell et al. ${ }^{2}$ showed that the inherent strength of human linea alba decreases towards the symphysis pubis with optimal security using bites of at least $1.2-1.5 \mathrm{~cm}$. Thus, it is doubtful whether their standard 6:1 SL:WL with a uniform 1 $\mathrm{cm}$ bite size can provide optimal closure in a tissue whose strength is non-uniform.

The genesis of midline incisional hernias is multifactorial, and we do need to elucidate the most effective technique for closing the abdomen. This may involve a standard SL:WL, but we must consider the differences of strength in using rectus sheath or linea alba, especially in the obese, and that the strength of the linea alba is non-uniform along the abdominal wall. Only then can we approach a correct formula.

\section{References}

1. Zollinger Jr RM, Zollinger RM. Laparotomy, the closure. In: Zollinger Jr RM, Zollinger RM. Atlas of Surgical Operation, 7th edn. New York: McGraw Hill, 1993; 26.

2. Campbell JA, Temple WJ, Frank CB, Huchcroft SA. A biomechanical study of suture pullout in linea alba. Surgery 1989; 106: 888-92.

Correspondence to: Dr RS Rampaul MB BS, Surgical Research Fellow, Professorial Unit of Surgery, City Hospital, Hucknall Road, Nottingham NG1 5PB, UK

Tel: +44 1159691169 ext. 46299; Fax: +44 115840 2632: E-mail mszrsr@msn1.surgery.nottingham.ac.uk 